\title{
RESSECÇÃO HEPÁTICA ROBÓTICA. Relato de experiência pioneira na América Latina
}

\author{
Marcel Autran C. MACHAD01', Fábio Ferrari MAKDISSI', \\ Rodrigo C. T. SURJAN ${ }^{1}$ e Ricardo Z. ABDALLA ${ }^{2}$
}

\begin{abstract}
RESUMO - Graças ao melhor conhecimento da anatomia segmentar do fígado e desenvolvimento de novas técnicas, houve aumento no número de indicações de hepatectomias. O desenvolvimento da cirurgia minimamente invasiva ocorreu paralelamente e o aumento da experiência, aliado ao desenvolvimento de novos instrumentais, resultaram no crescimento exponencial das ressecções hepáticas videolaparoscópicas. A abordagem laparoscópica pode tornar viável a ressecção hepática em pacientes cirróticos com hipertensão portal que não tolerariam este mesmo procedimento por via laparotômica. A cirurgia robótica surgiu nos últimos anos como a última fronteira de desenvolvimento técnico aplicado à videocirurgia. O presente trabalho descreve a experiência pioneira de ressecção hepática totalmente com o uso de robótica na América Latina, em paciente com carcinoma hepatocelular e cirrose hepática. A hepatectomia laparoscópica com o uso do sistema robótico Da Vinci permite refinamentos técnicos graças à visualização tridimensional do campo cirúrgico e utilização de instrumentais precisos e com grande amplitude de movimentação que simulam os movimentos da mão humana.
\end{abstract}

DESCRITORES - Hepatectomia. Hepatopatias, cirurgia. Carcinoma hepatocelular, cirurgia. Robótica. Laparoscopia.

\section{INTRODUÇÃO}

As ressecções hepáticas tornaram-se, nos últimos anos, o método de eleição para o tratamento curativo dos tumores hepáticos. Graças ao melhor conhecimento da anatomia segmentar do fígado e desenvolvimento de novas técnicas, houve aumento do número de indicações de hepatectomias. $\mathrm{O}$ desenvolvimento da cirurgia minimamente invasiva ocorreu paralelamente e o aumento da experiência, aliado ao desenvolvimento de novos instrumentais, resultaram no crescimento exponencial das ressecções hepáticas videolaparoscópicas ${ }^{(3,4,5)}$. A cirurgia robótica surgiu nos últimos anos como a última fronteira de desenvolvimento técnico aplicado à videocirurgia.

O objetivo do presente trabalho foi relatar a experiência pioneira de ressecção hepática totalmente com o uso de robótica na América Latina.

\section{MÉTODOS}

Paciente do sexo masculino com 72 anos de idade, com cirrose hepática criptogênica de classe funcional Child-Pugh A5. Durante seguimento apresentou piora súbita da função hepática com progressão para classe funcional B7. A ressonância magnética revelou nódulo suspeito para carcinoma hepatocelular em segmento 5 do fígado com $2 \mathrm{~cm}$ de diâmetro, sinais de hipertensão portal e redução do volume hepático. Endoscopia digestiva alta revelou varizes esofágicas de médio calibre. Foi indicado tratamento cirúrgico que consistiu de ressecção do segmento 5 com o uso de robótica. A intervenção cirúrgica foi realizada no dia 20 de agosto de 2008.

O sistema de robô empregado foi o da Vinci (Intuitive Surgical, Inc., Sunnyvale, CA, EUA). A técnica de hepatectomia robótica é semelhante à realizada por videolaparoscopia e previamente descrita pelos autores. Em resumo, o doente é colocado em posição supina, em decúbito dorsal horizontal. O primeiro trocarte é inserido pela técnica aberta. Pneumoperitônio com $\mathrm{CO}_{2}$ é estabelecido com pressão intra-abdominal de $12 \mathrm{~mm}$ $\mathrm{Hg}$. Foram utilizados quatro trocartes pelo cirurgião, dois de $11 \mathrm{~mm}$ e dois de $5 \mathrm{~mm}$. Um porto auxiliar, colocado na cicatriz umbilical, é utilizado pelo assistente para introduzir fios agulhados e aspirar o campo cirúrgico (Figura 1a). Uma vez posicionados os trocartes, o robô é posicionado e seus braços acoplados aos portos (Figura 1b). O cirurgião posiciona-se no console de comando do robô (Figura 1c). O controle dos braços é obtido com o uso dos dedos de maneira intuitiva (Figura 1d)

A intervenção iniciou-se com secção de aderências de cirurgia prévia (colecistectomia laparoscópica), seguida da secção do ligamento redondo e do ligamento falciforme, com utilização de bisturi harmônico. $\mathrm{O}$ hilo hepático foi circundado para eventual necessidade de controle vascular durante a hepatectomia.

Após a confirmação da localização do tumor no segmento 5 por ultrassonografia intraoperatória, foi realizada a demarcação da área a ser ressecada com bisturi

Departamento de Gastroenterologia, Faculdade de Medicina da Universidade de São Paulo; ${ }^{2}$ Centro de Treinamento de Cirurgia do Hospital Sírio Libanês, São Paulo, SP. Correspondência: Prof. Marcel Autran C. Machado - Rua Evangelista Rodrigues, 407 - 05463-000 - São Paulo, SP. E-mail: dr@drmarcel.com.br 


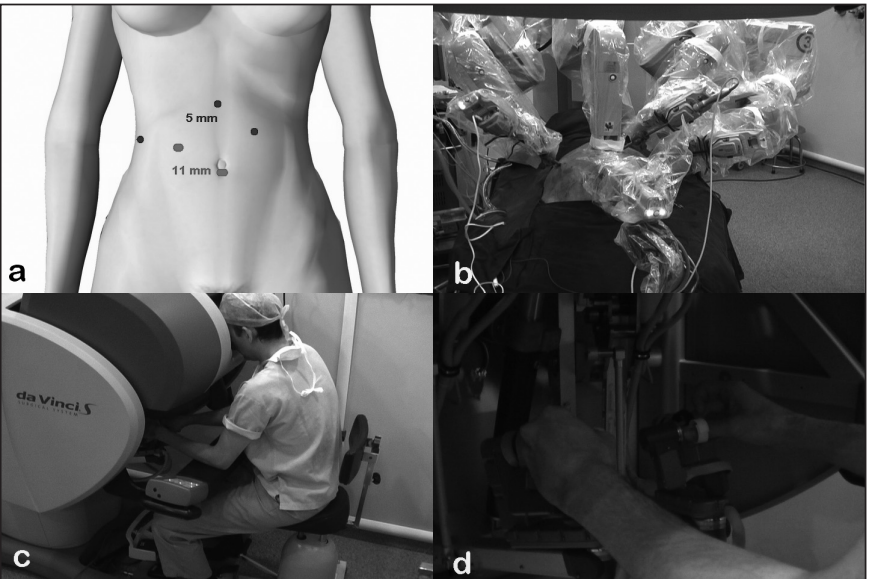

FIGURA 1. Hepatectomia robótica

a. Tipo e localização dos trocartes

b. Instalação do robô

c. Ergonomia do cirurgião no console

d. Ajuste das mãos e controle intuitivo das pinças

monopolar. A secção do parênquima hepático foi feita com combinação de bisturi bipolar e bisturi harmônico (Figura 2a). Vasos de médio calibre foram controlados com clipes metálicos e vasos de grande calibre foram suturados (Figura $2 b$ ).

O espécime cirúrgico foi colocado em saco plástico e retirado através do prolongamento do porto auxiliar umbilical (Figura 2c). A área cruenta é verificada para presença de fístula biliar e sangramento (Figura 2d).

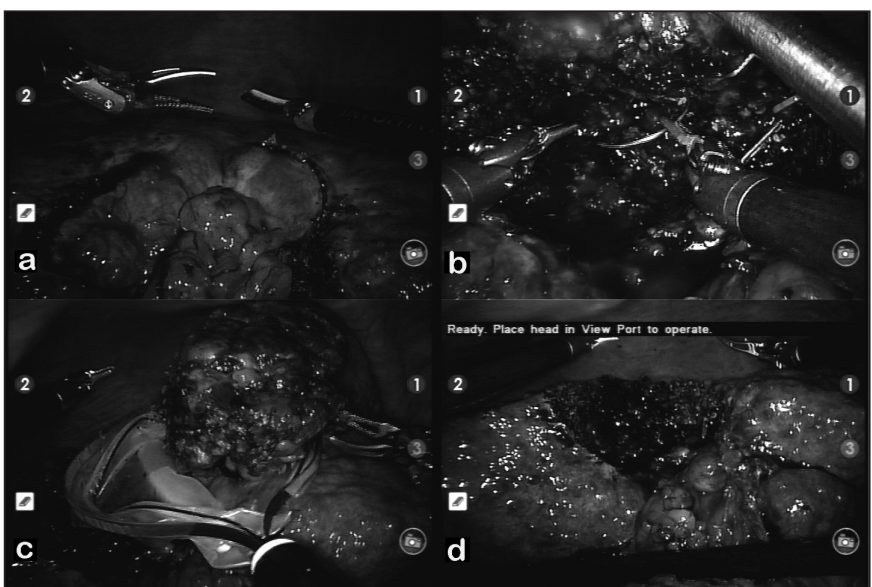

FIGURA 2. Hepatectomia robótica

a. Demarcação e início da secção do parênquima com bipolar e harmônico

b. Sutura é facilitada pela amplitude de movimentos das pinças robóticas

c. Retirada da peça cirúrgica protegida por saco plástico

d. Aspecto final após ressecção hepática do segmento 5

\section{RESULTADOS}

O tempo cirúrgico total foi de 2 horas. Não houve necessidade de manobra de Pringle. O sangramento foi mínimo e não houve necessidade de transfusão de hemoderivados. A cavidade abdominal não foi drenada. Não houve descompensação da função hepática no período pós-operatório e o paciente recebeu alta no $3^{\circ}$ dia de pós-operatório. O exame anatomopatológico demonstrou tratar-se de carcinoma hepatocelular moderadamente diferenciado e as margens cirúrgicas eram livres. $\mathrm{O}$ paciente apresenta-se bem e sem evidência de recidiva tumoral 4 meses após o procedimento.

\section{DISCUSSÃO}

A ressecção hepática por videolaparoscopia é método seguro e estabelecido no nosso meio ${ }^{(3,4,5)}$. A abordagem laparoscópica pode tornar viável a ressecção hepática em pacientes cirróticos com hipertensão portal, que não tolerariam este mesmo procedimento por via laparotômica ${ }^{(1)}$.

A cirurgia robótica nada mais é que uma videocirurgia assistida por robô. A robótica acrescenta algumas vantagens ao método puramente laparoscópico.

Com o sistema robótico, o cirurgião tem visão tridimensional real do campo cirúrgico e seu posicionamento sentado com os braços apoiados lhe confere ótima ergonomia (Figura 1c). Os instrumentos de cirurgia robótica foram especialmente desenvolvidos para conferir amplitude de movimentos ao cirurgião, semelhante ao da mão humana, e podendo atingir rotação de até $360^{\circ}$. A liberdade de movimento das pinças robóticas proporciona aprendizado rápido e intuitivo. O robô também pode ser ajustado para realizar movimentos com amplitude de escala reduzida, o que permite suturas em espaços pequenos com grande desenvoltura. Além disso, elimina tremores conferindo grande precisão de movimentos. A ressecção hepática por videolaparoscopia necessita frequentemente de grampeadores para controle de grandes vasos. O robô permite suturas com muita rapidez e agilidade, o que possibilita o controle de grandes vasos sem uso de grampeadores endoscópicos. No presente caso, o controle da veia hepática média foi feito por meio de sutura.

No console o cirurgião possui controle do posicionamento da câmera e de três braços automatizados. Por meio de pedais pode alternar o controle da câmera e dos três braços do robô. Assim a apresentação fica estática e não sujeita à fadiga ou falha do auxiliar. Nos momentos em que o cirurgião está utilizando os braços do robô, a câmera fica fixa e sem nenhum tremor. $\mathrm{O}$ terceiro braço tem a função de apresentação e, no paciente operado, foi utilizado um afastador de fígado.

Em países desenvolvidos, a cirurgia robótica tem suplantado as demais técnicas cirúrgicas como, por exemplo, a prostatectomia radical. No entanto, na literatura inglesa, os autores só encontraram um relato de ressecção hepática por robô ${ }^{(2)}$.

Os autores concluem que a hepatectomia laparoscópica com o uso do sistema robótico Da Vinci permite refinamentos técnicos graças à visualização tridimensional do campo cirúrgico e utilização de instrumentais precisos e com grande amplitude de movimentação que simulam os movimentos da mão humana. A desvantagem atual deste método consiste no custo ainda elevado, mas que tende a ser reduzido com sua maior utilização. Contribui para isto o fato deste método ter sido implementado na América Latina apenas recentemente, no início de 2008. 
Machado MAC, Makdissi FF, Surjan RCT, Abdalla RZ. First robotic-assisted laparoscopic liver resection in Latin America. Arq Gastroenterol. 2009;46(1): 78-80.

ABSTRACT - The surgical robotic system is superior to traditional laparoscopy in regards to 3-dimensional images and better instrumentations. Robotic surgery for hepatic resection has not yet been extensively reported. The aim of this paper is to report the first known case of liver resection with use of a computerassisted, or robotic, surgical device in Latin America. A 72-year-old male with cryptogenic liver cirrhosis and hepatocellular carcinoma was referred for surgical treatment. Preoperative clinical evaluation and laboratory data disclosed a Child-Pugh class A patient. Magnetic resonance imaging showed a $2.2 \mathrm{~cm}$ tumor in segment 5. Liver size was decreased and there were signs of portal hypertension, such as splenomegaly and enlarged portal vein collaterals. Preoperative upper digestive endoscopy disclosed esophageal varices. Five trocars were used. Liver transection was achieved with harmonic scalpel and bipolar forceps. Hemostasis of raw surface areas was accomplished with interrupted stitches. Operative time was 120 minutes. Blood loss was minimal and the patient did not receive transfusion. The recovery was uneventful and patient was discharged on the 3rd postoperative day without ascites formation. Laparoscopic hepatic resection can safely be performed. The laparoscopic approach may enable liver resection in patients with cirrhosis and evidence of liver failure that would contraindicate open surgery probably because it precludes the transection of major abdominal collaterals. The Da Vinci robotic system allowed for technical refinements of laparoscopic liver resection due to 3-dimensional visualization of the operative field and instruments with wrist-type end-effectors.

HEADINGS - Hepatectomy. Liver diseases, surgery. Carcinoma, hepatocellular, surgery. Robotics. Laparoscopy.

\section{REFERÊNCIAS}

1. Cherqui D, Laurent A, Tayar C, Chang S, Van Nhieu JT, Loriau J, Karoui M, Duvoux C, Dhumeaux D, Fagniez PL. Laparoscopic liver resection for peripheral hepatocellular carcinoma in patients with chronic liver disease: midterm results and perspectives. Ann Surg. 2006;243:499-506.

2. Choi SB, Park JS, Kim JK, Hyung WJ, Kim KS, Yoon DS, Lee WJ, Kim BR. Early experiences of robotic-assisted laparoscopic liver resection. Yonsei Med J. 2008;49:632-8.
3. Machado MA, Makdissi FF, Surjan RC, Teixeira AR, Bacchella T, Machado MC Hepatectomia direita por videolaparoscopia. Rev Col Bras Cir. 2007;34:189-92.

4. Machado MA, Makdissi FF, Galvão FH, Machado MC. Intrahepatic Glissonian approach for laparoscopic right segmental liver resections. Am J Surg. 2008;196:e38-e42.

5. Machado MA, Makdissi FF, Surjan RC, Oliveira AC, Pilla VF, Teixeira AR. Trisegmentectomia hepática direita por videolaparoscopia. Rev Col Bras Cir. 2008;35:338-41.

Recebido em 12/12/2008. Aprovado em 15/1/2009. 